\title{
Three-Dimensional Printing Assisted Preoperative Surgical Planning for Cerebral Arteriovenous Malformation
}

\author{
Inan Uzunoglu, ${ }^{1}$ Ceren Kızmazoglu, ${ }^{2}$ Resit Buğra Husemoglu, ${ }^{3}$ Gokhan Gurkan, ${ }^{1}$ Cansu Uzunoglu, ${ }^{4}$ Murat Atar, ${ }^{5}$ \\ Volkan Cakır, ${ }^{6}$ Hasan Emre Aydın, Murat Sayın, ${ }^{7}$ Nurullah Yuceer ${ }^{1}$ \\ Department of Neurosurgery, Katip Celebi Unı versity Ataturk Training and Research Hospital, Izmir, Turkey \\ Department of Neurosurgery, ${ }^{2}$ Dokuz Eylül University School of Medicine, Izmir, Turkey \\ Department of Biomechanic, ${ }^{3}$ Dokuz Eylül University School of Medicine, Izmir, Turkey \\ Department of Neurological Intensive Care, ${ }^{4}$ Ege University School of Medicine, Izmir, Turkey \\ Department of Neurosurgery, ${ }^{5}$ Sultan Abdulhamid Han Training and Research Hospital, Istanbul, Turkey \\ Department of Interventional Radiology, ${ }^{6}$ Tinaztepe University Galen Hospital, Izmir, Turkey \\ Department of Neurosurgery, ${ }^{7}$ Dumlupinar University Kutahya Evliya Celebi Training and Research Hospital, Kutahya, Turkey
}

Objective : The aim of this study to investigate the benefits of patient-based 3-dimensional (3D) cerebral arteriovenous malformation (AVM) models for preoperative surgical planning and education.

Methods : Fifteen patients were operated on for AVMs between 2015 and 2019 with patient-based 3D models. Ten patients' preoperative cranial angiogram screenings were evaluated preoperatively or perioperatively via patient-based 3D models. Two patients needed emergent surgical intervention; their models were solely designed based on their AVMs and used during the operation. However, the other patients who underwent elective surgery had the modeling starting from the skull base. These models were used both preoperatively and perioperatively. The benefits of patients arising from treatment with these models were evaluated via patient files and radiological data.

Results : Fifteen patients (10 males and five females) between 16 and 66 years underwent surgery. The mean age of the patients was $40.0 \pm 14.72$. The most frequent symptom patients observed were headaches. Four patients had intracranial bleeding; the symptom of admission was a loss of consciousness. Two patients (13.3\%) belonged to Spetzler-Martin (SM) grade I, four (26.7\%) belonged to SM grade II, eight (53.3\%) belonged to SM grade III, and one (6.7\%) belonged to SM grade IV. The mean operation duration was $3.44 \pm 0.47$ hours. Three patients (20\%) developed transient neurologic deficits postoperatively, whereas three other patients died (20\%).

Conclusion : Several technological innovations have emerged in recent years to reduce undesired outcomes and support the surgical team. For example, 3D models have been employed in various surgical procedures in the last decade. The routine usage of patient-based 3D models will not only support better surgical planning and practice, but it will also be useful in educating assistants and explaining the situation to the patient as well.

Key Words : Intracranial arteriovenous malformations · Three-dimensional printing · Spetzler-Martin grade.

- Received : January 20, 2021 •Revised : February 15, 2021 •Accepted : March 19, 2021

- Address for reprints : Inan Uzunoglu

Department of Neurosurgery, Katip Celebi University Ataturk Training and Research Hospital, Basın Sitesi, Karabaglar, Izmir 35360, Turkey

Tel : +90 53044180 91, Fax : +90 232243 15 30, E-mail : dr_inan_uzunoglu@hotmail.com, ORCID : https://orcid.org/0000-0001-5859-0443

This is an Open Access article distributed under the terms of the Creative Commons Attribution Non-Commercial License (http://creativecommons.org/licenses/by-nc/4.0) which permits unrestricted non-commercial use, distribution, and reproduction in any medium, provided the original work is properly cited. 


\section{INTRODUCTION}

Cerebral arteriovenous malformations (AVMs) are defined as folded vascular structures that do not have capillary vessels between the arteries and veins. AVMs are rare conditions and are believed to be responsible for $2 \%$ of all hemorrhagic strokes $^{13)}$. The annual risk of bleeding varies between $1 \%$ and $25 \%$ and causes hemorrhagic strokes in patients below 40 years of age. Patients are occasionally symptomatic after intracranial bleeding and seizure ${ }^{16)}$.

In cases with bleeding, the treatment of AVM via endovascular, microsurgical, or hybrid (embolization+microsurgery) approach is the generally accepted opinion. However, the debate on asymptomatic cases is still present. Although AVM resection was a cause of morbidity and mortality in high percentages before advancements in microsurgery, with the advancements in the last two decades, the applicability of microsurgery after rupture and the reduced relapse rates render microsurgery as the best surgical choice ${ }^{12)}$. The microsurgical approach is a safer option after the closure of all or some of the feeding arteries via the endovascular approach.

The development of 3-dimensional (3D) printers and their usage in medicine in the last decade has facilitated the modeling of anatomical structures. The widespread use of 3D printers in surgery has contributed to a better unveiling of the anatomical structures; therefore, it can be used to support patientdoctor communication and education of trainees ${ }^{15,19)} .3 \mathrm{D}$ modeling of vascular structures in neurosurgery started with aneurisms. Cerebral aneurysm surgery requires the surgeon to understand the complex 3D structures of aneurisms individually in addition to the precise information related to the main vessels and surrounding anatomical structures ${ }^{9)}$. Models support the surgeon to visually perceive the localization, size, and direction of an aneurism. Complex vascular structures and blood flow dynamics of AVMs are now easier to understand with these models, which have come into use for cerebral AVMs in recent years. Screenings obtained from patients angiograms through $3 \mathrm{D}$ reconstruction via recently used computer-based software can be interpreted differently based on personal experience. Additionally, revealing arterial and venous phases in these reconstructions is difficult and requires a broad clinical experience.

We determined blood flow dynamics by revealing arterial and venous phases because of the rapid and individual proto- typing of AVMs in the patients. Additionally, in this novel case series in the literature, we demonstrated that $3 \mathrm{D}$ printers eased the understanding of anatomical structures for the surgeon, which resulted in making the surgery safer and shorter.

\section{MATERIALS AND METHODS}

The Izmir Katip Celebi University non-interventional Ethics Committee provided their approval on August 28, 2019, with file decision number 394.

\section{Patient selection and surgical planning}

In this study, the patients with cerebral AVM, who applied to our clinic with various clinical symptoms between the years 2015 and 2019, underwent hybrid surgery. Patients were informed about the closure of the feeding arteries via the endovascular approach, and that they were going to have a microsurgical resection at the same session. In total, 15 patients were informed about the risks of the intervention, and their consent was obtained. Afterward, we planned their surgeries. Patients who refused interventions were not included in the study. After the endovascular intervention, patients were transported in intubated and sedated condition from the angiography unit to a neurosurgery operation room.

\section{Modeling with a three-dimensional printer}

Preoperative digital subtraction angiography (DSA) and 1-mm thick-sliced computed tomography angiography (Siemens Healthcare, Forchheim, Germany) scan starting from the skull base images of patients who were operated on due to cerebral AVM were saved to picture archiving and communication system (PACS) as digital imaging and communication in medicine (DICOM) data raw data obtained during rotational angiography. DSA automatically converted to DICOM by (Philips, North America Corporation, Arlington, TX, USA). The PACS images were sent to Health Sciences Institute Biomechanics Laboratory for their reconstruction into 3D images and the data were sliced by applying the threshold perception in $3 \mathrm{D}$ slicer software (3D slicer version 4.8; Isomics, Inc, Cambridge, MA, USA). These data were turned into a 3D model manually by a senior neurosurgeon and neuroradiologist after being transformed into a high-definition stereolithography (STL) format via 3D slicer software. Image pro- 
cessing was performed in the target region by using the STL file on 3D engineering software. Each STL file in separate skulls was uploaded to Meshmixer (Autodesk Inc., San Francisco, CA, USA), which is a software for STL, to be examined before developing the model. Then, they were sliced by Cura software (Ultimaker B.V., Utrecht, The Netherlands) to create G Codes. Process settings were standardized as following: extruder temperature at $215^{\circ} \mathrm{C}$, room temperature at $24^{\circ} \mathrm{C}$, the primary layer height of $0.1 \mathrm{~mm}$, and the filling rate of $20 \%$. 3D models of AVMs were printed on a 1: 1 scale, double-colored via Ultimaker 3 Extended (Ultimaker BV, Utrecht, The Netherlands), which is a double extruder desktop printer. Polylactic acid (PLA), which is a thermoplastic polyester, was used to extrude the plastic from the platform where it was hardened during the printing process. The printing process lasted in about 4-24 hours.

Two of the patients who underwent emergency surgery only had AVM modeling, whereas patients who underwent elective surgery had their modeling starting from the skull base, including the lesion. Although the models were examined during the operations in emergency cases, there were chances to study the models for more extended periods in elective cases. Models were transported to our hospital 15 minutes after printing.

\section{Collection of data}

Patients' data of symptoms, age, gender, Glasgow coma scale (GCS) at the time of admission, performed surgery, SpetzlerMartin (SM) grade of AVMs, benefits according to modified Rankin scale (mRS), operation duration, hospitalization duration, and follow-up duration were collected from the patients' files and radiological imaging system. The diameter of the internal carotid artery (ICA) and the diameter before the basilar artery bifurcations of models were revealed with these data and were compared with the diameter of similar anatomical structures measured from the $2 \mathrm{D}$ angiograms via a digital compass.

\section{Statistical analysis}

We used Statistical Package for the Social Sciences (SPSS version 15.0; IBM Corp., Armonk, NY, USA) for conducting statistical analysis in this study. Chi-square and Mann-Whitney $\mathrm{U}$ tests were used for the statistical evaluations. A $p$-value of less than 0.05 was considered statistically significant for this study.

\section{RESULTS}

In total, 15 patients between the ages of 16 and 66 years were operated on, of whom 10 were male and five were female. The mean age of our patients was $40.0 \pm 14.72$ years. Headache was the most frequent symptom and was present in eight of the

Table 1. Demographic, clinical, and radiology characteristics of patients with brain AVM

\begin{tabular}{|c|c|c|}
\hline & Hybrid approach, +/- & No. of patient \\
\hline \multicolumn{3}{|l|}{ Sex } \\
\hline Male & $8(53.4) / 2(13.3)$ & $10(66.7)$ \\
\hline Female & $5(33.3) / 0(0.0)$ & $5(33.3)$ \\
\hline \multicolumn{3}{|l|}{ Symptoms } \\
\hline Headache & $7(46.6) / 1(6.7)$ & $8(53.3)$ \\
\hline Consciousness & $3(20.0) / 2(13.3)$ & $5(33.3)$ \\
\hline Aphasia & 1 (6.6)/1 (6.6) & $2(13.3)$ \\
\hline Seizure & $1(6.7) / 0(0.0)$ & $1(6.7)$ \\
\hline Vertigo & $1(6.7) / 0(0.0)$ & $1(6.7)$ \\
\hline \multicolumn{3}{|l|}{ AVM size $(\mathrm{cm})$} \\
\hline $0-3$ & $2 / 1$ & 3 \\
\hline $3-6$ & $7 / 1$ & 8 \\
\hline$>6$ & $4 / 0$ & 4 \\
\hline \multicolumn{3}{|l|}{ Venous drainage } \\
\hline Superficial & $11 / 1$ & 12 \\
\hline Deep & $2 / 1$ & 3 \\
\hline \multicolumn{3}{|l|}{ Eloquence } \\
\hline Eloquent & $3(17.8) / 1(8.9)$ & $4^{*}(26.7)$ \\
\hline Noneloquent & $10(66.6) / 1(6.7)$ & $11(73.3)$ \\
\hline \multicolumn{3}{|l|}{ SM drade } \\
\hline 1 & 1 (6.6)/1 (6.6) & $2(13.3)$ \\
\hline 2 & $4(26.7) / 0(0.0)$ & $4(26.7)$ \\
\hline 3 & $7(46.6) / 1(6.7)$ & $8(53.3)$ \\
\hline 4 & $1(6.7) / 0(0.0)$ & $1(6.7)$ \\
\hline \multicolumn{3}{|l|}{ Hemorrhage } \\
\hline+ & $2(13.3) / 2(13.3)$ & $4(26.7)$ \\
\hline- & $11(73.3) / 0(0.0)$ & $11(73.3)$ \\
\hline \multicolumn{3}{|l|}{ GKS } \\
\hline$<8$ & 0 & $0(0.0)$ \\
\hline $9-13$ & $4(13.3) / 2(26.7)$ & $6(40.0)$ \\
\hline $14-15$ & $9(60.0) / 0(0.0)$ & $9(60.0)$ \\
\hline
\end{tabular}

Values are presented as number (\%). *Three of the AVM's were located in the posterior fossa and one in the motor area. AVM : arteriovenous malformation, SM : Spetzler-Martin, GKS : Glasgow coma scale 
patients (53.3\%). Four of our patients applied to our emergency service clinic due to hematoma after confusion. At the moment of application to the hospital, $60 \%$ of our patients' GCS was measured as 14-15 (Table 1).

Lesions of our patients were confirmed by preoperative angiogram and preoperative surgical observation (lesion diameter, eloquent region, and venous drainage) and classified according to SM AVM grade ${ }^{13)}$. A total of four (26.7\%) of the lesions were present in the eloquent region (Figs. 1 and 2), whereas $11(73.3 \%)$ of them were present in the non-eloquent region. The venous drainage of 12 patients (80\%) were superficial, and four patients (20\%) had deep venous drainage. Three patients (20\%) had a diameter between 0 and 3, eight patients (53.3\%) had a diameter between 3 and 6 , and four patients $(26.7 \%)$ had a diameter of 6 or wider length. As a result, eight patients (53.3\%) belonged to SM grade III, four patients (26.7\%) belonged to SM grade II (Figs. 3 and 4), two patients (13.3\%) belonged to SM grade I, and one patient belonged to SM grade IV (Table 1).

In total, 13 of the operated patients (86.7\%) underwent surgery after the embolization performed in our hospital's interventional radiology clinic. In eight patients (53.3\%), the feeding artery was clamped perioperatively (Table 2, Figs. 5 and 6).

Only one patient was presented with juvenile tonic clonic epilepsy (JTC). However, a total of three patients, including this one, experienced JTC during the early postoperative period. Suitable levetiracetam doses controlled the seizure. Apart from these three patients, two more patients revealed epileptiform symptoms on their electroencephalography, which were related to their lesion, but they did not have any seizures. The
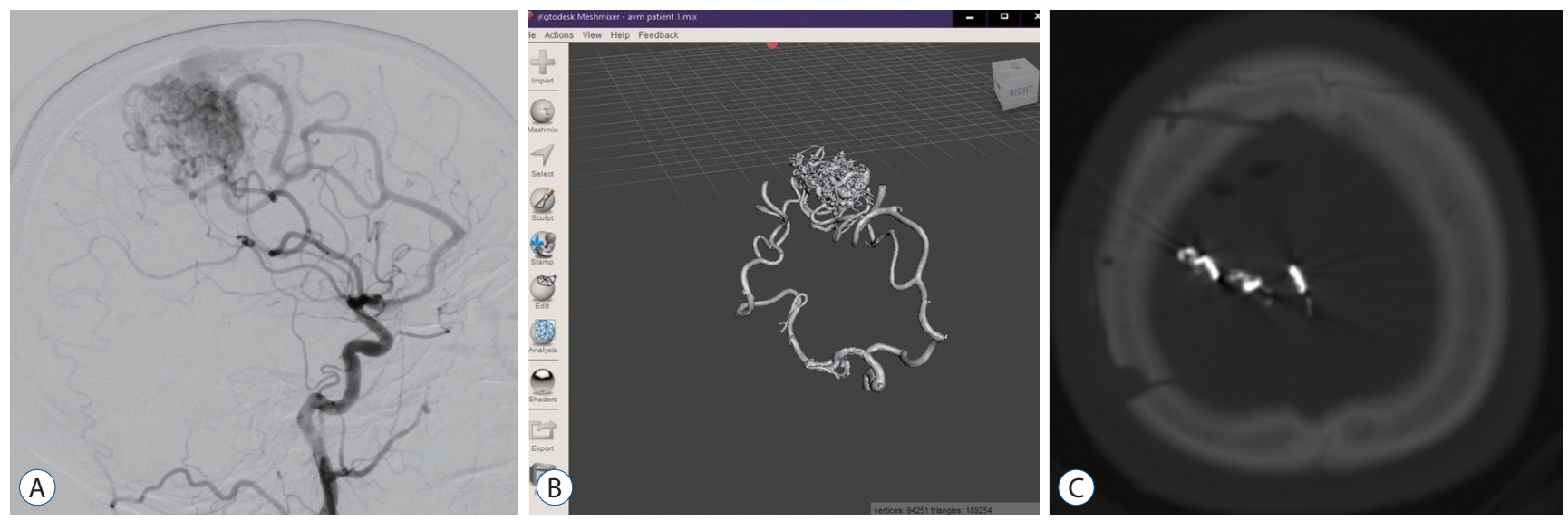

Fig. 1. A : Preoperative digital subtraction angiography image of 26 years female patient with feeding arteries from right medial cerebral artery and anterior cerebral artery. B : Anterior view of 3-dimensional solid in stereolithography format. C : Postoperative brain computed tomography of the patient.
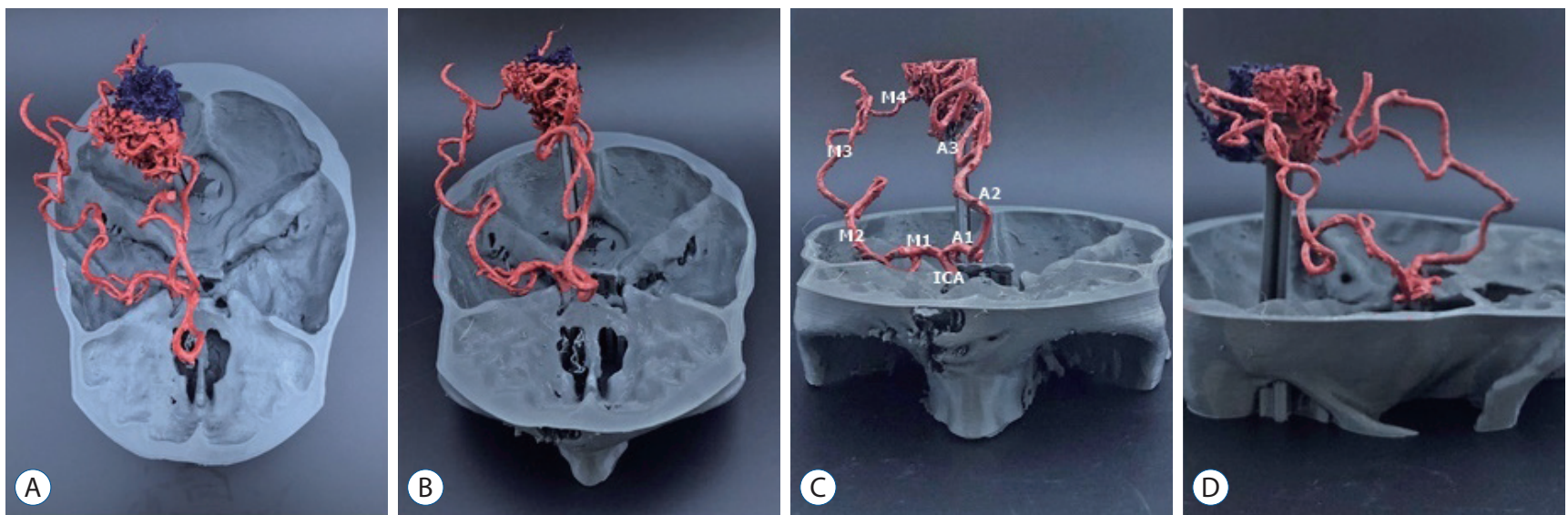

Fig. 2. Superior (A), superior anterior (B), anterior (C), and lateral (D) view of the printed brain arteriovenous malformation model with the skull (patient 1). $A_{1}, A_{2}$, and $A_{3}$ mean anterior cerebral artery branches. $M_{1}, M_{2}, M_{3}$, and $M_{4}$ mean middle cerebral artery branches. ICA : internal carotid artery. 

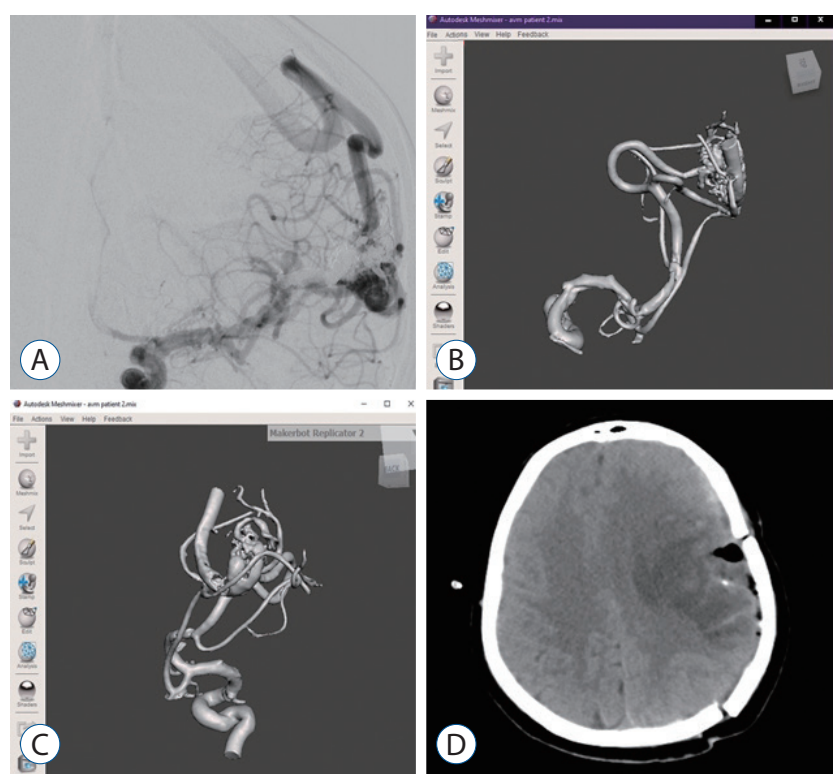

Fig. 3. A : Preoperative digital subtraction angiography image of 38 years female patient with feeding arteries from left medial cerebral artery. $B$ and C : Anterior-posterior view of 3-dimensional solid in stereolithography format. D : Postoperative brain computed tomography of the patient.
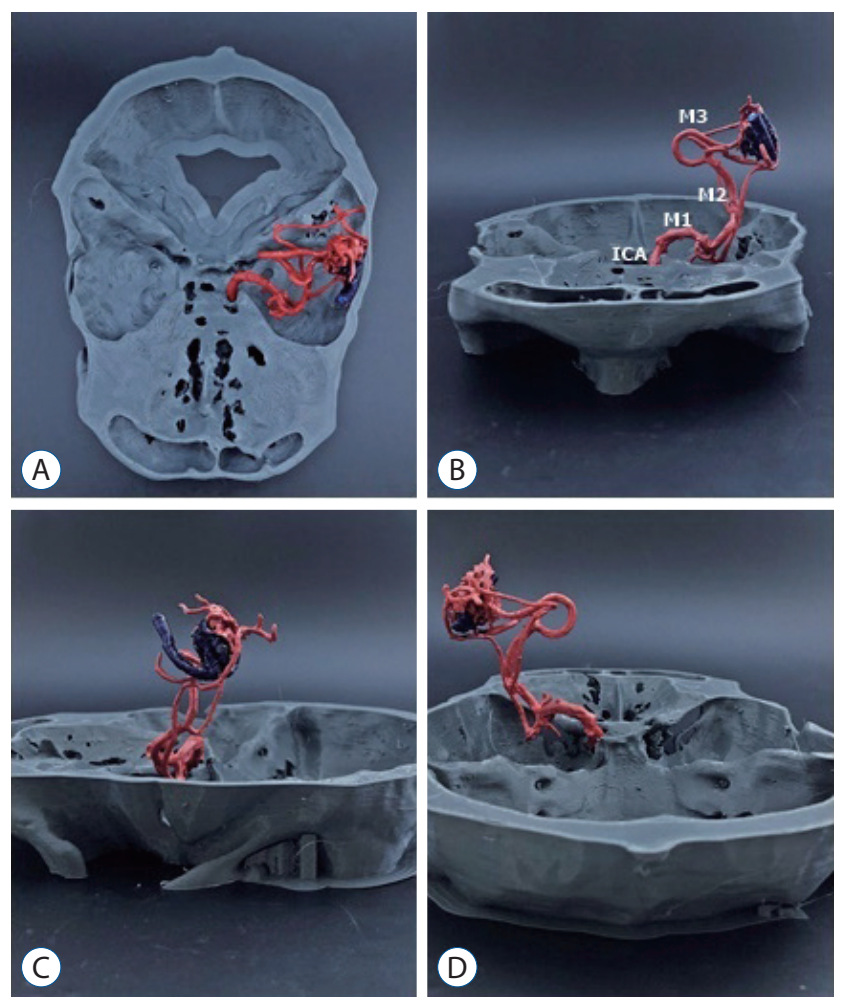

Fig. 4. Superior $(A)$, anterior $(B)$, lateral $(C)$, and posterior $(D)$ view of the printed brain arteriovenous malformation model with the skull (patient 2). $M_{1}, M_{2}$, and $M_{3}$ mean middle cerebral artery branches. ICA : internal carotid artery. maintenance of antiepileptic was recommended to all of our patients for the first year at the seizure blocking dose. Antiepileptic doses were arranged at the follow-up visits.

We reached to $95.2 \%$ of obliteration rate in postoperative lesions via hybrid intervention. In our study, the changes were examined by evaluating our patients' preoperative and postoperative general conditions with mRS (Table 2). The mean operation duration of patients was $3.44 \pm 0.47$ hours, whereas the mean hospitalization duration was $17.20 \pm 11.82$ days. Our patients' follow-up duration was $14.93 \pm 18.12$ months. Three of the operated patients developed transient neurologic deficits

Table 2. Perioperative and postoperative characteristics of patients

\begin{tabular}{ccc} 
& Hybrid approach, +/- & No. of patient \\
\hline Klipaj & & \\
Yes & $7(46.7) / 1(6.6)$ & $8(53.3)$ \\
No & $1(6.7) / 6(40.0)$ & $7(46.7)$
\end{tabular}

\begin{tabular}{|c|c|c|}
\hline \multicolumn{3}{|c|}{ Neurological deficit } \\
\hline Yes & $2(13.4) / 1^{*}(6.6)$ & $3(20.0)$ \\
\hline No & $11(73.3) / 1(6.7)$ & $12(80.0)$ \\
\hline
\end{tabular}

Preoperative $\mathrm{mRS}$

$\begin{array}{lll}0 & 9(60.0) / 0(0.0) & 9(60.0) \\ 1 & 0(0.0) / 0(0.0) & 0(0.0) \\ 2 & 1(6.7) / 0(0.0) & 1(6.7) \\ 3 & 1(6.7) / 0(0.0) & 1(6.7) \\ 4 & 1(6.7) / 2(13.4) & 3(20.0) \\ 5 & 1(6.7) / 0(0.0) & 1(6.7)\end{array}$

\begin{tabular}{lcc} 
Final mRS & & \\
0 & $5(33.3) / 2(13.4)$ & $7(46.7)$ \\
1 & $2(13.3) / 0(0.0)$ & $2(13.3)$ \\
2 & $1(6.7) / 0(0.0)$ & $1(6.7)$ \\
3 & $2(13.4) / 0(0.0)$ & $2(13.4)$ \\
4 & $0(0.0) / 0(0.0)$ & $0(0.0)$ \\
5 & $0(0.0) / 0(0.0)$ & $0(0.0)$ \\
6 (exitus) & $3(20.0)^{\dagger} / 0(0.0)$ & $3(20.0)$ \\
Obliteration rate (\%) & $96 / 90$ & $15(95.2)$ \\
Operation time (hours) & $3.42 / 3.60$ & $3.44 \pm 0.47(2.63-4.25)$ \\
Hospital stay (days) & $18.76 / 7.0$ & $17.20 \pm 11.82(5-45)$ \\
Follow-up times (months) & $14.15 / 20.00$ & $14.93 \pm 18.12(1-53)$ \\
\hline
\end{tabular}

Values are presented as mean \pm standard deviation (range) or number (\%). *Two of three patients with transient neurological deficits had frustrated hemiparesis and one had dysarthria. Deficits improved at 6 months followup. ${ }^{\dagger}$ While two of three patients with exitus developed meningitis, one patient died due to pneumonia. mRS : modified Rankin scale 
in the early postoperative period. Postoperatively, eight patients were discharged without any problems, whereas three patients died. Two out of the three mortalities developed meningitis, whereas one of them died due to pneumonia (Table 2).

Before the operation, the diameter of bilateral ICA and the diameter before basilar artery bifurcation were first measured with $2 \mathrm{D}$ models, and the results of these were compared with the diameter of the 3D models via a digital compass (Table 3).

\section{DISCUSSION}

The surgical treatment of cerebral AVMs is one of the most laborious procedures of neurosurgery. However, although the bleeding risk after a rapidly performed resection is set to zero, invasive interventions planned to be performed on patients during follow-up are not needed anymore ${ }^{3)}$.

With the advancements in medical screening techniques, the pathophysiology of brain vascular structures is revealed more clearly. The complexity of the vascular structure of the
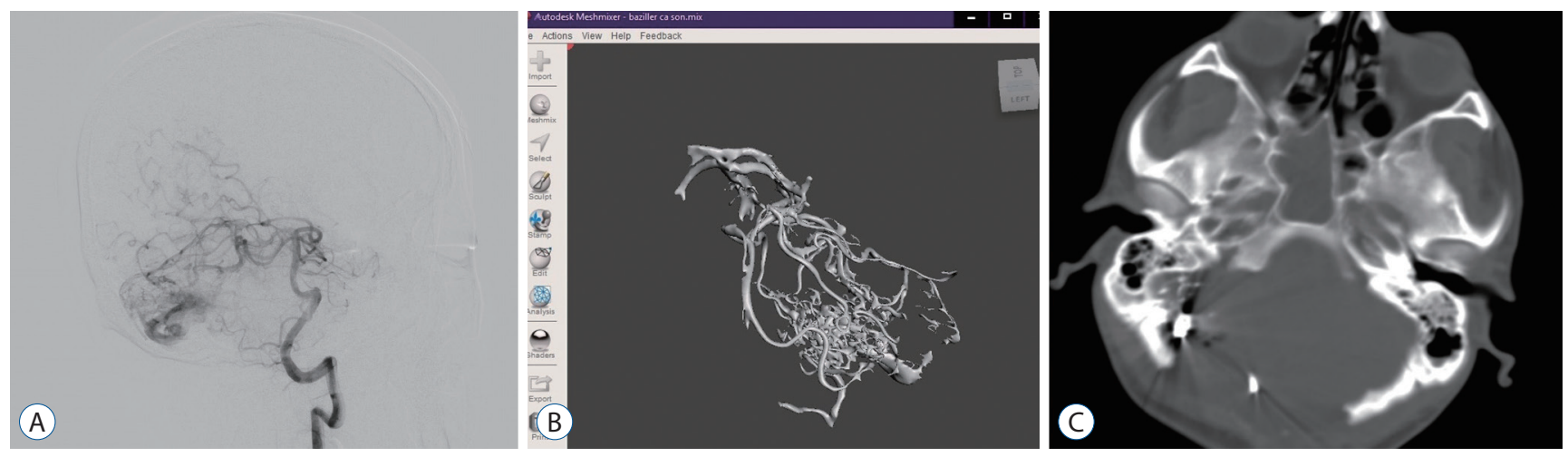

Fig. 5. A : Preoperative digital subtraction angiography image of 28 years male patient with feeding arteries from left superior cerebellar artery and posterior inferior cerebellar artery. B : Anterior view of 3-dimensional solid in stereolithography format. C : Postoperative brain computed tomography of the patient.
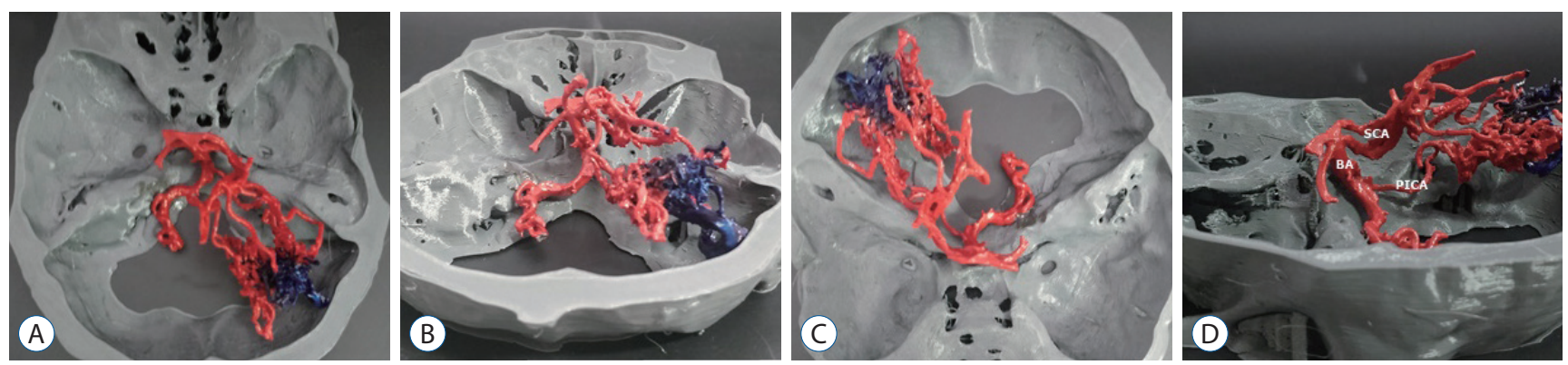

Fig. 6. Superior- posterior (A), posterior (B), superior-anterior (C), and lateral (D) view of the printed brain arteriovenous malformation model with the skull (patient 3). BA : basilar artery, SCA : superior cerebellar artery, PICA : posterior inferior cerebellar artery.

Table 3. Mean diameters of major arteries at angiography and 3-dimensional model ( $\mathrm{mm})$

\begin{tabular}{|c|c|c|c|c|c|c|c|}
\hline & \multicolumn{3}{|c|}{ Angiography } & \multicolumn{3}{|c|}{ Model } & \multirow{2}{*}{$p$-value } \\
\hline & Right ICA & Left ICA & Basilar artery & Right ICA & Left ICA & Basilar artery & \\
\hline Minimum & 3.00 & 2.56 & 2.48 & 3.82 & 3.28 & 3.37 & \\
\hline Maximum & 4.35 & 3.70 & 5.28 & 5.18 & 4.87 & 5.97 & \\
\hline Mean & 3.57 & 3.22 & 3.65 & 4.48 & 4.10 & 4.48 & 0.001 \\
\hline SD & 0.39 & 0.36 & 0.76 & 0.41 & 0.42 & 0.79 & \\
\hline
\end{tabular}

ICA : internal carotid artery, SD : standard deviation 
AVM and the inadequacy of 2D imaging has led to the emergence of 3D models for a better demonstration of the feeding artery, drainage vein, nidus, and positional relations of these structures. 3D modeling also provides the surgeon with a better chance to master and practice anatomical structures before and during the operation.

Patient-based models produced via 3D printers have revealed the feeding arteries and drainage veins of AVM in detail. However, because these models were produced with fused deposition modeling (FDM), nidus could not be revealed in detail. A part of the supporting materials was left around the nidus in order not to harm the small supporting arteries while cleaning the supporting materials. Nevertheless, this did not change our operative plan for any of our patients. Other 3D printer types, STLs, can print more detailed results, but the usage and consumables (resin) of these are more expensive for us than FDM. Therefore, we chose the FDM-type 3D printer because of its user-friendliness and cheap consumables.

Although cerebral AVMs occasionally occur in young and healthy individuals, it was reported that $50 \%$ of these patients were symptomatic after intracranial haemorrhage ${ }^{10,14)}$. In our case series, the mean age, $40 \pm 14.72$ years, was coherent with the literature. The number of patients applied with hemorrhage was four (26.7\%). It can be predicted that our small sample size caused this situation, and we only added the patients that we operated with the new technique. Epilepsy was revealed as the most frequent symptom of admission to the hospital after hemorrhage, and the symptom provides an incidental diagnosis in cerebral AVM cases ${ }^{18)}$. In our study, this rate was as low as 1/15 (6.7\%), which is different from the literature data. At the same time, electrophysiological epileptiform focus related to the location of the lesion, and surgical intervention was present in five of our patients (33.3\%). Postoperative seizures were controlled with suitable antiepileptic treatment. We confirmed that this rate was in correlation with the literature data ${ }^{18)}$.
The operation duration of cerebral AVMs operated via hybrid intervention was approximately 4.12 hours in a study conducted by Grüter et al. ${ }^{4}$, whereas this duration was 3.42 hours in our patients treated with both 3D printer support and hybrid technique. The routine usage of modeling with 3D printers is predicted to shorten the operation duration soon.

In a study conducted by Lawton et al. ${ }^{8)}$, it is revealed that mRS is better than Glasgow outcome scale in assessing the surgical benefits of patients; therefore, we followed our patients with mRS in our study. Research literature had shown that mRS was bettered in surgically treated hemorrhagic AVMs; however, it worsened in patients without hemorrhage. In our study, we found similar results, but they are not statistically significant (Table 4$)^{8}$. These results predict that a higher number of patients can reveal statistically similar data. Additionally, cerebral AVMs; obliteration treated with only microsurgery and hybrid intervention was similar, ${ }^{1,7,17,20)}$ with that in literature and resulted in 95.2\% (Table 2).

Research literature had reported that the morbidity rate in cerebral AVM surgery is approximately $16.6 \%{ }^{16)}$. Three of the patients $(20 \%)$ who operated in our clinic developed transient neurologic deficits, and these deficits were observed to regress in 6 months of follow-up. These three patients also underwent hybrid intervention. The rate in our case series is in parallel with the research literature. The mortality rate of operated patients with cerebral AVM was reported between $0 \%$ and $9 \%$ in previously conducted studies ${ }^{6-8,16)}$. In our study, this rate resulted in 20\% (3/15). Although the difference between mortality rates reveals that the support of the $3 \mathrm{D}$ printer does not provide any possible contribution to mortality rates, we believe that mortality rates might be in parallel with literature with increasing the subject number of the sample size.

Models of AVMs were printed after DSA. In these models, bilateral ICA and the diameter before basilar artery bifurcations were measured two-dimensionally from DSA image and 3D models via a digital compass. Statistically, a significant dif-

Table 4. Preoperative and postoperative mRS results of patients

\begin{tabular}{lcccc}
\hline & Preoperative mRS & Final mRS & Change & p-value \\
\hline Ruptured & 3.75 & 1.50 & 2.25 & 0.459 \\
Unruptured & 0.64 & 2.55 & -1.91 & 0.505 \\
Difference & 3.11 & -1.05 & 4.16 & \\
\hline
\end{tabular}

mRS : modified Rankin scale 
ference was detected between the two-dimensional angiogram measurements and 3D models ( $p=0.001$ ) (Table 3). The main reason for the difference between the artery diameters of patients was revealed as screening vessel walls, which were narrower than they were due to the accepting vessel images in DSA after contrast application source. However, it has been shown in the methodology that there can be an increase or decrease in vessel size in the smoothing process of creating a solid model in previous studies. This model's technician differs individually, as well. An experienced interventional neuroradiologist in our study measured DSA. To prevent any difference between models, the same technician and the same two neurosurgeons created the models. The standardization of our study was provided by this method. We achieved similar data to literature via the artery diameters of models printed in $3 \mathrm{D}$ printers ${ }^{2,5,11)}$. These results reveal that 3D models obtained similarly can be in the same size as the anatomical size, and they can be used conveniently in clinical studies (Table 3).

The ability of 3D models to be produced in short periods with millimetric precision and their usability in emergent cases such as cerebral AVM along with the development of a patient-based 3D model provide considerable advantages to the clinician. We wanted to report the series consisting of 15 patients of 4 years and show that the routine use of those models can help in achieving excellent results for both patient and surgical team. However, the fact that it is a brand new technology and costs 400 USD per patient is the biggest obstacle to its routine usage in the short run.

In our study, the brain parenchyma could not be modeled, since the vascular structures were modeled from DSA angiography images. 3D modeling of the brain parenchyma would bring both additional writing time and an additional cost. We hope that with the development and widespread use of 3D printers in the future, brain parenchyma can be printed more cheaply and in a shorter time with vascular sections. This will provide the clinician with more anatomical and surgical orientation.

In our study, the feeding artery and venous structures can be suitably mapped for printing with the help of the software; however, AVM nidus could not be modeled clearly due to its dense contrasting. Because of the type of printer, AVM modeling was made of PLA, and the materials that could show circulation more precisely were not used. Moreover, although the printing time has seen an incredible decline recently, detailed models can be printed in periods that are expected to be longer for AVM surgery. It is evident that if the printing duration can be decreased to minutes, then these models can be exploited more in emergent surgical patients. With all these limitations, 3D models, today and in the future, will help the surgical team, as well as increasing surgical surveys and decreasing morbidity.

\section{CONCLUSION}

We think that the routine use of modeling with 3D printers will provide a positive contribution to interventions conducted on the emergent patients and expertise on the surgical team. The routine usage of models can provide not only ease in surgical planning and practicing but also an opportunity for educating the assistants and explain the situation more clearly to patients in the following term.

\section{CONFLICTS OF INTEREST}

No potential conflict of interest relevant to this article was reported.

\section{INFORMED CONSENT}

Informed consent was obtained from all individual participants included in this study.

\section{AUTHOR CONTRIBUTIONS}

\author{
Conceptualization : CK, GG \\ Data curation : IU, GG \\ Formal analysis : VC, NY \\ Funding acquisition : CU, IU \\ Methodology : RBH, MA \\ Project administration : MA, MS \\ Visualization : HEA, RBH \\ Writing - original draft : MS, IU \\ Writing - review \& editing: NY, IU
}




\section{ORCID}

Inan Uzunoglu https://orcid.org/0000-0001-5859-0443

Ceren Kizmazoglu https://orcid.org/0000-0001-6146-0842

Resit Buğra Husemoglu https://orcid.org/0000-0003-1979-160X

Gokhan Gurkan https://orcid.org/0000-0003-1839-1014

Cansu Uzunoglu https://orcid.org/0000-0002-5969-3142

Murat Atar

https://orcid.org/0000-0001-6047-2956

Volkan Cakır

https://orcid.org/0000-0002-3039-8262

Hasan Emre Aydın https://orcid.org/0000-0002-8932-1542

Murat Sayın

https://orcid.org/0000-0001-8745-2656

Nurullah Yuceer https://orcid.org/0000-0003-3509-9939

\section{References}

1. Bervini $D$, Morgan MK, Ritson EA, Heller $G$ : Surgery for unruptured arteriovenous malformations of the brain is better than conservative management for selected cases: a prospective cohort study. J Neurosurg $121: 878-890,2014$

2. Burlakoti A, Kumaratilake J, Taylor J, Massy-Westropp N, Henneberg M : The cerebral basal arterial network: morphometry of inflow and outflow components. J Anat $230: 833-841,2017$

3. Davies JM, Yanamadala V, Lawton MT : Comparative effectiveness of treatments for cerebral arteriovenous malformations: trends in nationwide outcomes from 2000 to 2009. Neurosurg Focus 33 : E11, 2012

4. Grüter BE, Mendelowitsch I, Diepers M, Remonda L, Fandino J, Marbacher $\mathrm{S}$ : Combined endovascular and microsurgical treatment of arteriovenous malformations in the hybrid operating room. World Neurosurg 117 : e204-e214, 2018

5. Gunnal S, Farooqui M, Wabale R : Anatomical variability in the termination of the basilar artery in the human cadaveric brain. Turk Neurosurg 25 : 586-594, 2015

6. Heros RC, Tu YK : Unruptured arteriovenous malformations: a dilemma in surgical decision making. Clin Neurosurg 33 : 187-236, 1986

7. Jean WC, Huynh T, Tai AX, Felbaum DR, Syed HR, Ngo HM : Outcome of microsurgery for arteriovenous malformations in a resource-restricted environment: single-surgeon series from vietnam. World Neurosurg 132 : e66-e75, 2019

8. Lawton MT, Du R, Tran MN, Achrol AS, McCulloch CE, Johnston SC, et al. : Effect of presenting hemorrhage on outcome after microsurgical resection of brain arteriovenous malformations. Neurosurgery 56 : 485-493; discussion 485-493, 2005

9. Molyneux AJ, Kerr RS, Yu LM, Clarke M, Sneade M, Yarnold JA, et al. : International subarachnoid aneurysm trial (ISAT) of neurosurgical clipping versus endovascular coiling in 2143 patients with ruptured intracranial aneurysms: a randomised comparison of effects on survival, dependen$c y$, seizures, rebleeding, subgroups, and aneurysm occlusion. Lancet $366: 809-817,2005$

10. Potts MB, Lau D, Abla AA, Kim H, Young WL, Lawton MT, et al. : Current surgical results with low-grade brain arteriovenous malformations. J Neurosurg 122 : 912-920, 2015

11. Rhoton AL Jr : The supratentorial cranial space: microsurgical anatomy and surgical approaches. Neurosurgery 51 : S1-iii-S1-vi, 2002

12. Schramm J, Schaller K, Esche J, Boström A : Microsurgery for cerebral arteriovenous malformations: subgroup outcomes in a consecutive series of 288 cases. J Neurosurg 126 : 1056-1063, 2017

13. Spetzler RF, Martin NA : A proposed grading system for arteriovenous malformations. J Neurosurg 65 : 476-483, 1986

14. Starke RM, Komotar RJ, Hwang BY, Fischer LE, Garrett MC, Otten ML, et al. : Treatment guidelines for cerebral arteriovenous malformation microsurgery. Br J Neurosurg 23 : 376-386, 2009

15. Thawani JP, Pisapia JM, Singh N, Petrov D, Schuster JM, Hurst RW, et al. : Three-dimensional printed modeling of an arteriovenous malformation including blood flow. World Neurosurg 90 : 675-683.e2, 2016

16. Theofanis T, Chalouhi N, Dalyai R, Starke RM, Jabbour P, Rosenwasser $\mathrm{RH}$, et al. : Microsurgery for cerebral arteriovenous malformations: postoperative outcomes and predictors of complications in 264 cases. Neurosurg Focus 37 : E10, 2014

17. Tong X, Wu J, Cao Y, Zhao Y, Wang S, Zhao J : Microsurgical outcome of unruptured brain arteriovenous malformations: a single-center experience. World Neurosurg 99 : 644-655, 2017

18. von der Brelie C, Simon M, Esche J, Schramm J, Boström A : Seizure outcomes in patients with surgically treated cerebral arteriovenous malformations. Neurosurgery 77 : 762-768, 2015

19. Weinstock P, Prabhu SP, Flynn K, Orbach DB, Smith E : Optimizing cerebrovascular surgical and endovascular procedures in children via personalized 3D printing. J Neurosurg Pediatr 16 : 584-589, 2015

20. Wong J, Slomovic A, Ibrahim G, Radovanovic I, Tymianski M : Microsurgery for ARUBA trial (a randomized trial of unruptured brain arteriovenous malformation)-eligible unruptured brain arteriovenous malformations. Stroke 48 : 136-144, 2017 